PROCEEDINGS OF THE

AMERICAN MATHEMATICAL SOCIETY

Volume 126, Number 10, October 1998, Pages 2957-2968

S 0002-9939(98)04436-0

\title{
APPLICATIONS OF PSEUDO-MONOTONE OPERATORS WITH SOME KIND OF UPPER SEMICONTINUITY IN GENERALIZED QUASI-VARIATIONAL INEQUALITIES ON NON-COMPACT SETS
}

\author{
MOHAMMAD S. R. CHOWDHURY AND KOK-KEONG TAN
}

(Communicated by Dale Alspach)

\begin{abstract}
Let $E$ be a topological vector space and $X$ be a non-empty subset of $E$. Let $S: X \rightarrow 2^{X}$ and $T: X \rightarrow 2^{E^{*}}$ be two maps. Then the generalized quasi-variational inequality (GQVI) problem is to find a point $\hat{y} \in S(\hat{y})$ and a point $\hat{w} \in T(\hat{y})$ such that $\operatorname{Re}\langle\hat{w}, \hat{y}-x\rangle \leq 0$ for all $x \in S(\hat{y})$. We shall use Chowdhury and Tan's 1996 generalized version of Ky Fan's minimax inequality as a tool to obtain some general theorems on solutions of the GQVI on a paracompact set $X$ in a Hausdorff locally convex space where the set-valued operator $T$ is either strongly pseudo-monotone or pseudo-monotone and is upper semicontinuous from $\operatorname{co}(A)$ to the weak*-topology on $E^{*}$ for each nonempty finite subset $A$ of $X$.
\end{abstract}

\section{INTRODUCTION}

If $X$ is a set, we shall denote by $2^{X}$ the family of all non-empty subsets of $X$ and by $\mathcal{F}(X)$ the family of all non-empty finite subsets of $X$. Let $E$ be a topological vector space. We shall denote by $E^{*}$ the continuous dual of $E$, by $\langle w, x\rangle$ the pairing between $E^{*}$ and $E$ for $w \in E^{*}$ and $x \in E$ and by $R e\langle w, x\rangle$ the real part of $\langle w, x\rangle$. If $X \subset E, S: X \rightarrow 2^{X}$ and $T: X \rightarrow E^{*}$, the quasi-variational inequality problem (QVI) is to find a point $\hat{y} \in S(\hat{y})$ such that $\operatorname{Re}\langle T(\hat{y}), \hat{y}-x\rangle \leq 0$ for all $x \in S(\hat{y})$. The QVI was first introduced by Bensousson and Lions in 1973 (see, e.g., [2]) in connection with impulse control. Again, if we consider a set-valued map $T: X \rightarrow 2^{E^{*}}$, then the generalized quasi-variational inequality problem (GQVI) is to find a point $\hat{y} \in S(\hat{y})$ and a point $\hat{w} \in T(\hat{y})$ such that $R e\langle\hat{w}, \hat{y}-x\rangle \leq 0$ for all $x \in S(\hat{y})$. The GQVI was introduced by Chan and Pang [4] in 1982 if $E=\mathbb{R}^{n}$ and by Shih and Tan [11] in 1985 if $E$ is infinite dimensional.

In this paper, we shall use Chowdhury and Tan's generalized version [5, Theorem 2] of Ky Fan's minimax inequality [8, Theorem 1] as a tool to obtain some

Received by the editors May 15, 1996 and, in revised form, March 7, 1997.

1991 Mathematics Subject Classification. Primary 47H04, 47H05, 47H09, 47H10; Secondary 49J35, 49J40, 54C60.

Key words and phrases. Generalized quasi-variational inequality, locally convex space, partition of unity, paracompact sets, lower semi-continuous, upper semi-continuous, strongly pseudomonotone, pseudo-monotone and monotone operators.

The work of the second author was partially supported by NSERC of Canada under grant A-8096.

(C)1998 American Mathematical Society 
general theorems on solutions of the GQVI on a paracompact set $X$ in a locally convex Hausdorff topological vector space where the set-valued operator $T$ is strongly pseudo-monotone or pseudo-monotone and is upper semicontinuous from $\operatorname{co}(A)$ to the weak*-topology on $E^{*}$ for each $A \in \mathcal{F}(X)$.

We shall use our following set-valued generalization of the classical pseudomonotone operator. The classical definition of a pseudo-monotone operator was introduced by Brézis, Nirenberg and Stampacchia in [3]. For a slightly general definition of a pseudo-monotone operator we refer to [5, Definition 1].

Definition 1.1. Let $E$ be a topological vector space, $X$ be a non-empty subset of $E$ and $T: X \rightarrow 2^{E^{*}}$. If $h: X \rightarrow \mathbb{R}$, then $T$ is said to be (1) $h$-pseudomonotone if for each $y \in X$ and every net $\left\{y_{\alpha}\right\}_{\alpha \in \Gamma}$ in $X$ converging to $y$ with $\lim \sup _{\alpha}\left[\inf _{u \in T\left(y_{\alpha}\right)} \operatorname{Re}\left\langle u, y_{\alpha}-y\right\rangle+h\left(y_{\alpha}\right)-h(y)\right] \leq 0$, we have

$\liminf _{\alpha}\left[\inf _{u \in T\left(y_{\alpha}\right)} \operatorname{Re}\left\langle u, y_{\alpha}-x\right\rangle+h\left(y_{\alpha}\right)-h(x)\right] \geq \inf _{w \in T(y)} \operatorname{Re}\langle w, y-x\rangle+h(y)-h(x)$

for all $x \in X$; (2) pseudo-monotone if $T$ is $h$-pseudo-monotone with $h \equiv 0$.

\section{Generalized quasi-Variational ineQualities FOR STRONGLY PSEUDO-MONOTONE OPERATORS}

In this section we shall introduce the notion of strongly pseudo-monotone operators and obtain some general theorems on solutions of the GQVI on paracompact sets in locally convex Hausdorff topological vector spaces.

We shall begin with the following:

Definition 2.1. Let $E$ be a topological vector space, $X$ be a non-empty subset of $E$ and $T: X \rightarrow 2^{E^{*}}$. If $h: X \rightarrow \mathbb{R}$, then $T$ is said to be (1) strongly h-pseudomonotone if for each continuous function $\theta: X \rightarrow[0,1]$, for each $y \in X$ and every net $\left\{y_{\alpha}\right\}_{\alpha \in \Gamma}$ in $X$ converging to $y$ with

$$
\limsup _{\alpha}\left[\theta\left(y_{\alpha}\right)\left(\inf _{u \in T\left(y_{\alpha}\right)} \operatorname{Re}\left\langle u, y_{\alpha}-y\right\rangle+h\left(y_{\alpha}\right)-h(y)\right)\right] \leq 0
$$

we have

$$
\begin{array}{r}
\limsup _{\alpha}\left[\theta\left(y_{\alpha}\right)\left(\inf _{u \in T\left(y_{\alpha}\right)} \operatorname{Re}\left\langle u, y_{\alpha}-x\right\rangle+h\left(y_{\alpha}\right)-h(x)\right)\right] \\
\geq\left[\theta(y)\left(\inf _{w \in T(y)} \operatorname{Re}\langle w, y-x\rangle+h(y)-h(x)\right)\right]
\end{array}
$$

for all $x \in X$; (2) strongly pseudo-monotone if $T$ is strongly $h$-pseudo-monotone with $h \equiv 0$.

Clearly, every strongly pseudo-monotone operator is also a pseudo-monotone operator as defined in [5].

Proposition 2.1. Let $X$ be a non-empty subset of a topological vector space $E$. If $T: X \rightarrow E^{*}$ is monotone and continuous from the relative weak topology on $X$ to the weak* topology on $E^{*}$, then $T$ is strongly pseudo-monotone.

Proof. Let us consider any arbitrary continuous function $\theta: X \rightarrow[0,1]$. Suppose $\left\{y_{\alpha}\right\}_{\alpha \in \Gamma}$ is a net in $X$ and $y \in X$ with $y_{\alpha} \rightarrow y$ (and

$$
\left.\limsup _{\alpha}\left[\theta\left(y_{\alpha}\right)\left(\operatorname{Re}\left\langle T y_{\alpha}, y_{\alpha}-y\right\rangle\right)\right] \leq 0\right) .
$$


Then for any $x \in X$ and $\epsilon>0$, there are $\beta_{1}, \beta_{2} \in \Gamma$ with $\left|\theta\left(y_{\alpha}\right) \operatorname{Re}\left\langle T y, y_{\alpha}-y\right\rangle\right|<\frac{\epsilon}{2}$ for all $\alpha \geq \beta_{1}$ and $\left|\theta\left(y_{\alpha}\right) \operatorname{Re}\left\langle T y_{\alpha}-T y, y-x\right\rangle\right|<\frac{\epsilon}{2}$ for all $\alpha \geq \beta_{2}$. Choose $\beta_{0} \in \Gamma$ with $\beta_{0} \geq \beta_{1}, \beta_{2}$. Thus

$$
\begin{aligned}
& \theta\left(y_{\alpha}\right) \operatorname{Re}\left\langle T y_{\alpha}, y_{\alpha}-x\right\rangle=\theta\left(y_{\alpha}\right) \operatorname{Re}\left\langle T y_{\alpha}, y_{\alpha}-y\right\rangle+\theta\left(y_{\alpha}\right) \operatorname{Re}\left\langle T y_{\alpha}, y-x\right\rangle \\
& \geq \theta\left(y_{\alpha}\right) \operatorname{Re}\left\langle T y, y_{\alpha}-y\right\rangle+\theta\left(y_{\alpha}\right) \operatorname{Re}\left\langle T y_{\alpha}, y-x\right\rangle \\
& =\theta\left(y_{\alpha}\right) \operatorname{Re}\left\langle T y, y_{\alpha}-y\right\rangle+\theta\left(y_{\alpha}\right) \operatorname{Re}\left\langle T y_{\alpha}-T y, y-x\right\rangle+\theta\left(y_{\alpha}\right) \operatorname{Re}\langle T y, y-x\rangle \\
& >-\frac{\epsilon}{2}-\frac{\epsilon}{2}+\theta\left(y_{\alpha}\right) \operatorname{Re}\langle T y, y-x\rangle \text { for all } \alpha \geq \beta_{0}
\end{aligned}
$$

so that $\inf _{\alpha \geq \beta_{0}} \theta\left(y_{\alpha}\right) \operatorname{Re}\left\langle T y_{\alpha}, y_{\alpha}-x\right\rangle \geq-\epsilon+\inf _{\alpha \geq \beta_{0}} \theta\left(y_{\alpha}\right) \operatorname{Re}\langle T y, y-x\rangle$. It follows that $\lim _{\sup } \theta\left(y_{\beta}\right) \operatorname{Re}\left\langle T y_{\beta}, y_{\beta}-x\right\rangle \geq \liminf _{\beta} \theta\left(y_{\beta}\right) \operatorname{Re}\left\langle T y_{\beta}, y_{\beta}-x\right\rangle \geq-\epsilon+$ $\theta(y) \operatorname{Re}\langle T y, y-x\rangle$. As $\epsilon>0$ is arbitrary,

$$
\limsup _{\beta} \theta\left(y_{\beta}\right) \operatorname{Re}\left\langle T y_{\beta}, y_{\beta}-x\right\rangle \geq \theta(y) \operatorname{Re}\langle T y, y-x\rangle \text {. }
$$

Hence $T$ is strongly pseudo-monotone.

We shall now establish the following result:

Theorem 2.1. Let $E$ be a locally convex Hausdorff topological vector space, $X$ be a non-empty paracompact convex subset of $E$ and $h: E \rightarrow \mathbb{R}$ be convex. Let $S: X \rightarrow 2^{X}$ be upper semicontinuous such that each $S(x)$ is compact convex and $T: X \rightarrow 2^{E^{*}}$ be strongly h-pseudo-monotone and be upper semicontinuous from $\operatorname{co}(A)$ to the weak*-topology on $E^{*}$ for each $A \in \mathcal{F}(X)$ such that each $T(x)$ is weak ${ }^{*}$-compact convex. Suppose that the set

$$
\Sigma=\left\{y \in X: \sup _{x \in S(y)}\left[\inf _{w \in T(y)} \operatorname{Re}\langle w, y-x\rangle+h(y)-h(x)\right]>0\right\}
$$

is open in $X$. Suppose further that there exist a non-empty compact subset $K$ of $X$ and a point $x_{0} \in X$ such that $x_{0} \in K \cap S(y)$ and $\inf _{w \in T(y)} \operatorname{Re}\left\langle w, y-x_{0}\right\rangle+h(y)-$ $h\left(x_{0}\right)>0$ for all $y \in X \backslash K$. Then there exists $\hat{y} \in K$ such that (i) $\hat{y} \in S(\hat{y})$ and (ii) there exists $\hat{w} \in T(\hat{y})$ with $\operatorname{Re}\langle\hat{w}, \hat{y}-x\rangle \leq h(x)-h(\hat{y})$ for all $x \in S(\hat{y})$.

Proof. We divide the proof into two steps:

Step 1 . There exists a point $\hat{y} \in X$ such that $\hat{y} \in S(\hat{y})$ and

$$
\sup _{x \in S(\hat{y})}\left[\inf _{w \in T(\hat{y})} \operatorname{Re}\langle w, \hat{y}-x\rangle+h(\hat{y})-h(x)\right] \leq 0 .
$$

Suppose the contrary. Then for each $y \in X$, either $y \notin S(y)$ or there exists $x \in S(y)$ such that $\inf _{w \in T(y)} \operatorname{Re}\langle w, y-x\rangle+h(y)-h(x)>0$; that is, $y \notin S(y)$ or $y \in \Sigma$. If $y \notin S(y)$, then by Hahn-Banach separation theorem, there exists $p \in E^{*}$ such that $\operatorname{Re}\langle p, y\rangle-\sup _{x \in S(y)} \operatorname{Re}\langle p, x\rangle>0$. For each $y \in X$, set $\gamma(y):=$ $\sup _{x \in S(y)}\left[\inf _{w \in T(y)} \operatorname{Re}\langle w, y-x\rangle+h(y)-h(x)\right]$. Let $V_{0}:=\{y \in X \mid \gamma(y)>0\}=\Sigma$ and for each $p \in E^{*}$, set $V_{p}:=\left\{y \in X: \operatorname{Re}\langle p, y\rangle-\sup _{x \in S(y)} \operatorname{Re}\langle p, x\rangle>0\right\}$.

Then $X=V_{0} \cup \bigcup_{p \in E^{*}} V_{p}$. Since each $V_{p}$ is open in $X$ by Lemma 1 in [11] and $V_{0}$ is open in $X$ by hypothesis, $\left\{V_{0}, V_{p}: p \in E^{*}\right\}$ is an open covering for $X$. Since $X$ is paracompact, there is a continuous partition of unity $\left\{\beta_{0}, \beta_{p}: p \in E^{*}\right\}$ for $X$ subordinated to the open cover $\left\{V_{0}, V_{p}: p \in E^{*}\right\}$ (see, e.g., Theorem VIII.4.2 of Dugundji in [7]); that is, for each $p \in E^{*}, \beta_{p}: X \rightarrow[0,1]$ and $\beta_{0}: X \rightarrow[0,1]$ are continuous functions such that for each $p \in E^{*}, \beta_{p}(y)=0$ for all $y \in X \backslash V_{p}$ and $\beta_{0}(y)=0$ for all $y \in X \backslash V_{0}$ and $\left\{\right.$ support $\beta_{0}$, support $\left.\beta_{p}: p \in E^{*}\right\}$ is locally finite and $\beta_{0}(y)+\sum_{p \in E^{*}} \beta_{p}(y)=1$ for each $y \in X$. Note that for each $A \in \mathcal{F}(X), h$ is 
continuous on $\operatorname{co}(A)$ (see e.g. [10, Corollary 10.1.1, p.83]). Define $\phi: X \times X \rightarrow \mathbb{R}$ by

$$
\phi(x, y)=\beta_{0}(y)\left[\min _{w \in T(y)} \operatorname{Re}\langle w, y-x\rangle+h(y)-h(x)\right]+\sum_{p \in E^{*}} \beta_{p}(y) \operatorname{Re}\langle p, y-x\rangle
$$

for each $x, y \in X$. Then we have the following.

(1) Since $E$ is Hausdorff, for each $A \in \mathcal{F}(X)$ and each fixed $x \in \operatorname{co}(A)$, the map $y \mapsto \min _{w \in T(y)} \operatorname{Re}\langle w, y-x\rangle+h(y)-h(x)$ is lower semicontinuous on $c o(A)$ by Lemma 3 in [5] and the fact that $h$ is continuous on $\operatorname{co}(A)$ and therefore the map $y \mapsto \beta_{0}(y)\left[\min _{w \in T(y)} \operatorname{Re}\langle w, y-x\rangle+h(y)-h(x)\right]$ is lower semicontinuous on $\operatorname{co}(A)$ by Lemma 3 in [12]. Also for each fixed $x \in X, y \mapsto \sum_{p \in E^{*}} \beta_{p}(y) \operatorname{Re}\langle p, y-x\rangle$ is continuous on $X$. Hence, for each $A \in \mathcal{F}(X)$ and each fixed $x \in \operatorname{co}(A)$, the map $y \longmapsto \phi(x, y)$ is lower semicontinuous on $c o(A)$.

(2) For each $A \in \mathcal{F}(X)$ and for each $y \in c o(A), \min _{x \in A} \phi(x, y) \leq 0$. Indeed, if this were false, then for some $A=\left\{x_{1}, \cdots, x_{n}\right\} \in \mathcal{F}(X)$ and some $y \in \operatorname{co}(A)$ (say $y=\sum_{i=1}^{n} \lambda_{i} x_{i}$ where $\lambda_{1}, \cdots, \lambda_{n} \geq 0$ with $\sum_{i=1}^{n} \lambda_{i}=1$ ), we have $\min _{1 \leq i \leq n} \phi\left(x_{i}, y\right)>0$. Then for each $i=1, \cdots, n$,

$$
\beta_{0}(y)\left[\min _{w \in T(y)} \operatorname{Re}\left\langle w, y-x_{i}\right\rangle+h(y)-h\left(x_{i}\right)\right]+\sum_{p \in E^{*}} \beta_{p}(y) \operatorname{Re}\left\langle p, y-x_{i}\right\rangle>0
$$

so that

$$
\begin{gathered}
0=\phi(y, y)=\beta_{0}(y)\left[\min _{w \in T(y)} \operatorname{Re}\left\langle w, y-\sum_{i=1}^{n} \lambda_{i} x_{i}\right\rangle+h(y)-h\left(\sum_{i=1}^{n} \lambda_{i} x_{i}\right)\right] \\
+\sum_{p \in E^{*}} \beta_{p}(y) \operatorname{Re}\left\langle p, y-\sum_{i=1}^{n} \lambda_{i} x_{i}\right\rangle \\
\geq \sum_{i=1}^{n} \lambda_{i}\left(\beta_{0}(y)\left[\min _{w \in T(y)} \operatorname{Re}\left\langle w, y-x_{i}\right\rangle+h(y)-h\left(x_{i}\right)\right]\right. \\
\left.+\sum_{p \in E^{*}} \beta_{p}(y) \operatorname{Re}\left\langle p, y-x_{i}\right\rangle\right)>0,
\end{gathered}
$$

which is a contradiction.

(3) Suppose $A \in \mathcal{F}(X), x, y \in \operatorname{co}(A)$ and $\left\{y_{\alpha}\right\}_{\alpha \in \Gamma}$ is a net in $X$ converging to $y$ with $\phi\left(t x+(1-t) y, y_{\alpha}\right) \leq 0$ for all $\alpha \in \Gamma$ and all $t \in[0,1]$.

Then for $t=0$ we have $\phi\left(y, y_{\alpha}\right) \leq 0$ for all $\alpha \in \Gamma$, i.e.,

$$
\beta_{0}\left(y_{\alpha}\right)\left[\min _{w \in T\left(y_{\alpha}\right)} R e\left\langle w, y_{\alpha}-y\right\rangle+h\left(y_{\alpha}\right)-h(y)\right]+\sum_{p \in E^{*}} \beta_{p}\left(y_{\alpha}\right) \operatorname{Re}\left\langle p, y_{\alpha}-y\right\rangle \leq 0
$$

for all $\alpha \in \Gamma$. Hence

$$
\begin{aligned}
\limsup _{\alpha}\left[\beta_{0}\left(y_{\alpha}\right)\right. & \left.\left(\min _{w \in T\left(y_{\alpha}\right)} \operatorname{Re}\left\langle w, y_{\alpha}-y\right\rangle+h\left(y_{\alpha}\right)-h(y)\right)\right] \\
& +\liminf _{\alpha}\left(\sum_{p \in E^{*}} \beta_{p}\left(y_{\alpha}\right) \operatorname{Re}\left\langle p, y_{\alpha}-y\right\rangle\right) \\
\leq \limsup _{\alpha} & {\left[\beta_{0}\left(y_{\alpha}\right)\left(\min _{w \in T\left(y_{\alpha}\right)} \operatorname{Re}\left\langle w, y_{\alpha}-y\right\rangle+h\left(y_{\alpha}\right)-h(y)\right)\right.} \\
& \left.+\sum_{p \in E^{*}} \beta_{p}\left(y_{\alpha}\right) \operatorname{Re}\left\langle p, y_{\alpha}-y\right\rangle\right] \leq 0 .
\end{aligned}
$$


Therefore $\lim \sup _{\alpha}\left[\beta_{0}\left(y_{\alpha}\right)\left(\min _{w \in T\left(y_{\alpha}\right)} \operatorname{Re}\left\langle w, y_{\alpha}-y\right\rangle+h\left(y_{\alpha}\right)-h(y)\right)\right] \leq 0$. Since $T$ is strongly $h$-pseudo-monotone, we have

$$
\begin{gathered}
{\left[\lim \sup _{\alpha}\left[\beta_{0}\left(y_{\alpha}\right)\left(\min _{w \in T\left(y_{\alpha}\right)} \operatorname{Re}\left\langle w, y_{\alpha}-x\right\rangle+h\left(y_{\alpha}\right)-h(x)\right)\right]\right.} \\
\quad \geq \beta_{0}(y)\left(\min _{w \in T(y)} \operatorname{Re}\langle w, y-x\rangle+h(y)-h(x)\right) .
\end{gathered}
$$

Thus

$$
\begin{gathered}
\operatorname{lim\operatorname {sup}}\left[\beta_{0}\left(y_{\alpha}\right)\left(\min _{w \in T\left(y_{\alpha}\right)} \operatorname{Re}\left\langle w, y_{\alpha}-x\right\rangle+h\left(y_{\alpha}\right)-h(x)\right)\right]+\sum_{p \in E^{*}} \beta_{p}(y) \operatorname{Re}\langle p, y-x\rangle \\
\geq \beta_{0}(y)\left(\min _{w \in T(y)} \operatorname{Re}\langle w, y-x\rangle+h(y)-h(x)\right)+\sum_{p \in E^{*}} \beta_{p}(y) \operatorname{Re}\langle p, y-x\rangle .
\end{gathered}
$$

For $t=1$ we have $\phi\left(x, y_{\alpha}\right) \leq 0$ for all $\alpha \in \Gamma$, i.e.,

$$
\beta_{0}\left(y_{\alpha}\right)\left[\min _{w \in T\left(y_{\alpha}\right)} \operatorname{Re}\left\langle w, y_{\alpha}-x\right\rangle+h\left(y_{\alpha}\right)-h(x)\right]+\sum_{p \in E^{*}} \beta_{p}\left(y_{\alpha}\right) \operatorname{Re}\left\langle p, y_{\alpha}-x\right\rangle \leq 0
$$

for all $\alpha \in \Gamma$. Therefore

$$
\begin{aligned}
\limsup _{\alpha}\left[\beta_{0}\left(y_{\alpha}\right)\right. & \left.\left(\min _{w \in T\left(y_{\alpha}\right)} \operatorname{Re}\left\langle w, y_{\alpha}-x\right\rangle+h\left(y_{\alpha}\right)-h(x)\right)\right] \\
& +\liminf _{\alpha}\left[\sum_{p \in E^{*}} \beta_{p}\left(y_{\alpha}\right) \operatorname{Re}\left\langle p, y_{\alpha}-x\right\rangle\right] \\
\leq \limsup _{\alpha} & {\left[\beta_{0}\left(y_{\alpha}\right)\left(\min _{w \in T\left(y_{\alpha}\right)} \operatorname{Re}\left\langle w, y_{\alpha}-x\right\rangle+h\left(y_{\alpha}\right)-h(x)\right)\right.} \\
& \left.+\sum_{p \in E^{*}} \beta_{p}\left(y_{\alpha}\right) \operatorname{Re}\left\langle p, y_{\alpha}-x\right\rangle\right] \leq 0 .
\end{aligned}
$$

Thus

$$
\begin{gathered}
\limsup _{\alpha}\left[\beta_{0}\left(y_{\alpha}\right)\left(\min _{w \in T\left(y_{\alpha}\right)} \operatorname{Re}\left\langle w, y_{\alpha}-x\right\rangle+h\left(y_{\alpha}\right)-h(x)\right)\right] \\
+\sum_{p \in E^{*}} \beta_{p}(y) \operatorname{Re}\langle p, y-x\rangle \leq 0 .
\end{gathered}
$$

Hence by $(2.1)$ and $(2.2)$, we have $\phi(x, y) \leq 0$.

(4) By hypothesis, there exist a non-empty compact (and therefore closed) subset $K$ of $X$ and a point $x_{0} \in X$ such that $x_{0} \in K \cap S(y)$ and $\inf _{w \in T(y)} \operatorname{Re}\left\langle w, y-x_{0}\right\rangle+$ $h(y)-h\left(x_{0}\right)>0$ for each $y \in X \backslash K$. Thus for each $y \in X \backslash K$,

$$
\beta_{0}(y)\left[\inf _{w \in T(y)} \operatorname{Re}\left\langle w, y-x_{0}\right\rangle+h(y)-h\left(x_{0}\right)\right]>0
$$

whenever $\beta_{0}(y)>0$ and $\operatorname{Re}\left\langle p, y-x_{0}\right\rangle>0$ whenever $\beta_{p}(y)>0$ for $p \in E^{*}$. Consequently,

$$
\begin{aligned}
\phi\left(x_{0}, y\right)= & \beta_{0}(y)\left[\inf _{w \in T(y)} \operatorname{Re}\left\langle w, y-x_{0}\right\rangle+h(y)-h\left(x_{0}\right)\right] \\
& +\sum_{p \in E^{*}} \beta_{p}(y) \operatorname{Re}\left\langle p, y-x_{0}\right\rangle>0
\end{aligned}
$$

for all $y \in X \backslash K$. 
Then $\phi$ satisfies all hypotheses of Theorem 2 in [5]. Hence by Theorem 2 in [5], there exists a point $\hat{y} \in K$ such that $\phi(x, \hat{y}) \leq 0$ for all $x \in X$; i.e.,

$$
\beta_{0}(\hat{y})\left[\inf _{w \in T(\hat{y})} R e\langle w, \hat{y}-x\rangle+h(\hat{y})-h(x)\right]+\sum_{p \in E^{*}} \beta_{p}(\hat{y}) \operatorname{Re}\langle p, \hat{y}-x\rangle \leq 0
$$

for all $x \in X$.

If $\gamma(\hat{y})=0$, choose any $\hat{x} \in S(\hat{y})$; if $\gamma(\hat{y})>0$, choose any $\hat{x} \in S(\hat{y})$ such that $\inf _{w \in T(\hat{y})} \operatorname{Re}\langle w, \hat{y}-\hat{x}\rangle+h(\hat{y})-h(\hat{x}) \geq \frac{\gamma(\hat{y})}{2}>0$.

If $\beta_{0}(\hat{y})>0$, then $\hat{y} \in V_{0}=\Sigma$ so that $\gamma(\hat{y})>0$; it follows that

$$
\beta_{0}(\hat{y})\left[\inf _{w \in T(\hat{y})} R e\langle w, \hat{y}-\hat{x}\rangle+h(\hat{y})-h(\hat{x})\right]>0 .
$$

If $\beta_{p}(\hat{y})>0$ for some $p \in E^{*}$, then $\hat{y} \in V_{p}$ and hence $\operatorname{Re}\langle p, \hat{y}\rangle>\sup _{x \in S(\hat{y})} \operatorname{Re}\langle p, x\rangle$ $\geq \operatorname{Re}\langle p, \hat{x}\rangle$ so that $\operatorname{Re}\langle p, \hat{y}-\hat{x}\rangle>0$. Then note that $\beta_{p}(\hat{y}) \operatorname{Re}\langle p, \hat{y}-\hat{x}\rangle>0 \quad$ whenever $\beta_{p}(\hat{y})>0$ for $p \in E^{*}$.

Since $\beta_{0}(\hat{y})>0$ or $\beta_{p}(\hat{y})>0$ for some $p \in E^{*}$, it follows that

$$
\phi(\hat{x}, \hat{y})=\beta_{0}(\hat{y})\left[\inf _{w \in T(\hat{y})} \operatorname{Re}\langle w, \hat{y}-\hat{x}\rangle+h(\hat{y})-h(\hat{x})\right]+\sum_{p \in E^{*}} \beta_{p}(\hat{y}) \operatorname{Re}\langle p, \hat{y}-\hat{x}\rangle>0,
$$

which contradicts (2.3). This contradiction proves Step 1.

Step 2. There exists a point $\hat{w} \in T(\hat{y})$ such that $\operatorname{Re}\langle\hat{w}, \hat{y}-x\rangle+h(\hat{y})-h(x) \leq$ 0 for all $x \in S(\hat{y})$.

Note that for each fixed $x \in S(\hat{y}), w \mapsto R e\langle w, \hat{y}-x\rangle+h(\hat{y})-h(x)$ is convex and continuous on $T(\hat{y})$ and for each fixed $w \in T(\hat{y}), x \mapsto R e\langle w, \hat{y}-x\rangle+h(\hat{y})-h(x)$ is concave on $S(\hat{y})$. Thus by Kneser's Minimax Theorem in [9] (see also Aubin [1, pp.40-41]), we have

$$
\begin{aligned}
& \min _{w \in T(\hat{y})} \max _{x \in S(\hat{y})}[\operatorname{Re}\langle w, \hat{y}-x\rangle+h(\hat{y})-h(x)] \\
& =\max _{x \in S(\hat{y})} \min _{w \in T(\hat{y})}[\operatorname{Re}\langle w, \hat{y}-x\rangle+h(\hat{y})-h(x)] .
\end{aligned}
$$

Hence $\min _{w \in T(\hat{y})} \max _{x \in S(\hat{y})}[R e\langle w, \hat{y}-x\rangle+h(\hat{y})-h(x)] \leq 0$ by Step 1. Since $T(\hat{y})$ is compact, there exists $\hat{w} \in T(\hat{y})$ such that $\operatorname{Re}\langle\hat{w}, \hat{y}-x\rangle+h(\hat{y})-h(x) \leq 0 \quad$ for all $x \in$ $S(\hat{y})$.

If $X$ is compact, we obtain the following immediate consequence of Theorem 2.1:

Theorem 2.2. Let $E$ be a locally convex Hausdorff topological vector space, $X$ be a non-empty compact convex subset of $E$ and $h: E \rightarrow \mathbb{R}$ be convex. Let $S: X \rightarrow 2^{X}$ be upper semicontinuous such that each $S(x)$ is closed convex and $T: X \rightarrow 2^{E^{*}}$ be strongly $h$-pseudo-monotone and be upper semicontinuous from co $(A)$ to the weak ${ }^{*}$ topology on $E^{*}$ for each $A \in \mathcal{F}(X)$ such that each $T(x)$ is weak $k^{*}$ compact convex. Suppose the set $\Sigma=\left\{y \in X: \sup _{x \in S(y)}\left[\inf _{w \in T(y)} \operatorname{Re}\langle w, y-x\rangle+h(y)-h(x)\right]>0\right\}$ is open in $X$. Then there exists $\hat{y} \in X$ such that (i) $\hat{y} \in S(\hat{y})$ and (ii) there exists $\hat{w} \in T(\hat{y})$ with $\operatorname{Re}\langle\hat{w}, \hat{y}-x\rangle \leq h(x)-h(\hat{y})$ for all $x \in S(\hat{y})$.

Note that if $X$ is also bounded in Theorem 2.1, the map $S: X \rightarrow 2^{X}$ is, in addition, lower semicontinuous and for each $y \in \Sigma, T$ is upper semicontinuous at $y$ in $X$, then the set $\Sigma$ in Theorem 2.1 is always open in $X$ as can be seen in the proof of the following: 
Theorem 2.3. Let $E$ be a locally convex Hausdorff topological vector space, $X$ be a non-empty paracompact convex and bounded subset of $E$ and $h: E \rightarrow \mathbb{R}$ be convex. Let $S: X \rightarrow 2^{X}$ be continuous such that each $S(x)$ is compact convex and $T: X \rightarrow 2^{E^{*}}$ be strongly $h$-pseudo-monotone and be upper semicontinuous from $\operatorname{co}(A)$ to the weak* $k^{*}$ topology on $E^{*}$ for each $A \in \mathcal{F}(X)$ such that each $T(x)$ is weak* $k^{*}$ compact convex. Suppose that for each

$$
y \in \Sigma=\left\{y \in X: \sup _{x \in S(y)}\left[\inf _{w \in T(y)} \operatorname{Re}\langle w, y-x\rangle+h(y)-h(x)\right]>0\right\},
$$

$T$ is upper semicontinuous at $y$ from the relative topology on $X$ to the strong topology on $E^{*}$. Suppose further that there exist a non-empty compact subset $K$ of $X$ and a point $x_{0} \in X$ such that $x_{0} \in K \cap S(y)$ and

$$
\inf _{w \in T(y)} \operatorname{Re}\left\langle w, y-x_{0}\right\rangle+h(y)-h\left(x_{0}\right)>0
$$

for all $y \in X \backslash K$. Then there exists $\hat{y} \in K$ such that (i) $\hat{y} \in S(\hat{y})$ and (ii) there exists $\hat{w} \in T(\hat{y})$ with $\operatorname{Re}\langle\hat{w}, \hat{y}-x\rangle \leq h(x)-h(\hat{y})$ for all $x \in S(\hat{y})$.

Proof. By virtue of Theorem 2.1, we need only show that the set

$$
\Sigma:=\left\{y \in X: \sup _{x \in S(y)}\left[\inf _{w \in T(y)} R e\langle w, y-x\rangle+h(y)-h(x)\right]>0\right\}
$$

is open in $X$. Indeed, let $y_{0} \in \Sigma$; then there exists $x_{0} \in S\left(y_{0}\right)$ such that $\alpha:=$ $\inf _{w \in T\left(y_{0}\right)} R e\left\langle w, y_{0}-x_{0}\right\rangle+h\left(y_{0}\right)-h\left(x_{0}\right)>0$.

Let $W:=\left\{w \in E^{*}: \sup _{z_{1}, z_{2} \in X}\left|\left\langle w, z_{1}-z_{2}\right\rangle\right|<\frac{\alpha}{6}\right\}$. Then $W$ is a strongly open neighborhood of 0 in $E^{*}$ so that $U_{1}:=T\left(y_{0}\right)+W$ is an open neighborhood of $T\left(y_{0}\right)$ in $E^{*}$. Since $T$ is upper semicontinuous at $y_{0}$ in $X$, there exists an open neighborhood $N_{1}$ of $y_{0}$ in $X$ such that $T(y) \subset U_{1}$ for all $y \in N_{1}$.

Now, the rest of the proof is similar to the proof of Theorem 2.2 in [6]. Hence by the rest of the proof of Theorem 2.2 in [6], $\Sigma$ is open in $X$. This proves the theorem.

If $X$ is compact, we obtain the following immediate consequence of Theorem 2.3:

Theorem 2.4. Let $E$ be a locally convex Hausdorff topological vector space, $X$ be a non-empty compact convex subset of $E$ and $h: E \rightarrow \mathbb{R}$ be convex. Let $S: X \rightarrow 2^{X}$ be continuous such that each $S(x)$ is closed convex and $T: X \rightarrow 2^{E^{*}}$ be strongly $h$ pseudo-monotone and be upper semicontinuous from $\operatorname{co}(A)$ to the weak $k^{*}$-topology on $E^{*}$ for each $A \in \mathcal{F}(X)$ such that each $T(x)$ is weak ${ }^{*}$-compact convex. Suppose that for each $y \in \Sigma=\left\{y \in X: \sup _{x \in S(y)}\left[\inf _{w \in T(y)} \operatorname{Re}\langle w, y-x\rangle+h(y)-h(x)\right]>0\right\}, T$ is upper semicontinuous at $y$ from the relative topology on $X$ to the strong topology on $E^{*}$. Then there exists $\hat{y} \in X$ such that (i) $\hat{y} \in S(\hat{y})$ and (ii) there exists $\hat{w} \in T(\hat{y})$ with $\operatorname{Re}\langle\hat{w}, \hat{y}-x\rangle \leq h(x)-h(\hat{y})$ for all $x \in S(\hat{y})$.

We remark here that in Theorems 2.1-2.4, the condition " $h: E \rightarrow \mathbb{R}$ be convex" can be replaced by the condition " $h: X \rightarrow \mathbb{R}$ be convex such that $\left.h\right|_{c o(A)}$ is continuous for each $A \in \mathcal{F}(X)$ ".

\section{Generalized quasi-Variational inequalities FOR PSEUDO-MONOTONE OPERATORS}

In this section we shall obtain some existence theorems of generalized quasivariational inequalities for pseudo-monotone operators (Definition 1.1) on paracompact convex sets. 
We shall first establish the following result:

Theorem 3.1. Let $E$ be a locally convex Hausdorff topological vector space, $X$ be a non-empty paracompact convex and bounded subset of $E$ and $h: E \rightarrow \mathbb{R}$ be convex such that $h(X)$ is bounded. Let $S: X \rightarrow 2^{X}$ be upper semicontinuous such that each $S(x)$ is compact convex and $T: X \rightarrow 2^{E^{*}}$ be h-pseudo-monotone and be upper semicontinuous from $\operatorname{co}(A)$ to the weak*-topology on $E^{*}$ for each $A \in \mathcal{F}(X)$ such that each $T(x)$ is weak ${ }^{*}$-compact convex and $T(X)$ is strongly bounded. Suppose that the set $\Sigma=\left\{y \in X: \sup _{x \in S(y)}\left[\inf _{w \in T(y)} \operatorname{Re}\langle w, y-x\rangle+h(y)-h(x)\right]>0\right\}$ is open in $X$. Suppose further that there exist a non-empty compact subset $K$ of $X$ and a point $x_{0} \in X$ such that $x_{0} \in K \cap S(y)$ and $\inf _{w \in T(y)} R e\left\langle w, y-x_{0}\right\rangle+h(y)-h\left(x_{0}\right)>0$ for all $y \in X \backslash K$. Then there exists $\hat{y} \in K$ such that (i) $\hat{y} \in S(\hat{y})$ and (ii) there exists $\hat{w} \in T(\hat{y})$ with $\operatorname{Re}\langle\hat{w}, \hat{y}-x\rangle \leq h(x)-h(\hat{y})$ for all $x \in S(\hat{y})$.

Proof. We divide the proof into two steps:

Step 1 . There exists a point $\hat{y} \in X$ such that $\hat{y} \in S(\hat{y})$ and

$$
\sup _{x \in S(\hat{y})}\left[\inf _{w \in T(\hat{y})} \operatorname{Re}\langle w, \hat{y}-x\rangle+h(\hat{y})-h(x)\right] \leq 0 .
$$

Suppose the contrary. Then for each $y \in X$, either $y \notin S(y)$ or there exists $x \in S(y)$ such that $\inf _{w \in T(y)} \operatorname{Re}\langle w, y-x\rangle+h(y)-h(x)>0$; that is, $y \notin S(y)$ or $y \in \Sigma$. If $y \notin S(y)$, then by Hahn-Banach separation theorem, there exists $p \in E^{*}$ such that $\operatorname{Re}\langle p, y\rangle-\sup _{x \in S(y)} \operatorname{Re}\langle p, x\rangle>0$. For each $y \in X$, set $\gamma(y):=$ $\sup _{x \in S(y)}\left[\inf _{w \in T(y)} \operatorname{Re}\langle w, y-x\rangle+h(y)-h(x)\right]$. Let $V_{0}:=\{y \in X \mid \gamma(y)>0\}=\Sigma$ and for each $p \in E^{*}$, set $V_{p}:=\left\{y \in X: \operatorname{Re}\langle p, y\rangle-\sup _{x \in S(y)} \operatorname{Re}\langle p, x\rangle>0\right\}$.

Then $X=V_{0} \cup \bigcup_{p \in E^{*}} V_{p}$. Since each $V_{p}$ is open in $X$ by Lemma 1 in [11] and $V_{0}$ is open in $X$ by hypothesis, $\left\{V_{0}, V_{p}: p \in E^{*}\right\}$ is an open covering for $X$. Since $X$ is paracompact, there is a continuous partition of unity $\left\{\beta_{0}, \beta_{p}: p \in E^{*}\right\}$ for $X$ subordinated to the open cover $\left\{V_{0}, V_{p}: p \in E^{*}\right\}$. Note that for each $A \in \mathcal{F}(X), h$ is continuous on $c o(A)$ (see e.g. [10, Corollary 10.1.1, p.83]). Define $\phi: X \times X \rightarrow \mathbb{R}$ by

$$
\phi(x, y)=\beta_{0}(y)\left[\min _{w \in T(y)} \operatorname{Re}\langle w, y-x\rangle+h(y)-h(x)\right]+\sum_{p \in E^{*}} \beta_{p}(y) \operatorname{Re}\langle p, y-x\rangle
$$

for each $x, y \in X$. Then we have the following.

(1) The same argument in proving (1) in the proof of Theorem 2.1 shows that for each $A \in \mathcal{F}(X)$ and each fixed $x \in c o(A)$, the map $y \longmapsto \phi(x, y)$ is lower semicontinuous on $\operatorname{co}(A)$.

(2) The same argument in proving (2) in the proof of Theorem 2.1 shows that for each $A \in \mathcal{F}(X)$ and for each $y \in c o(A), \min _{x \in A} \phi(x, y) \leq 0$.

(3) Suppose $A \in \mathcal{F}(X), x, y \in c o(A)$ and $\left\{y_{\alpha}\right\}_{\alpha \in \Gamma}$ is a net in $X$ converging to $y$ with $\phi\left(t x+(1-t) y, y_{\alpha}\right) \leq 0$ for all $\alpha \in \Gamma$ and all $t \in[0,1]$.

Case 1. $\beta_{0}(y)=0$.

Note that $\beta_{0}\left(y_{\alpha}\right) \geq 0$ for each $\alpha \in \Gamma$ and $\beta_{0}\left(y_{\alpha}\right) \rightarrow 0$. Since $T(X)$ is strongly bounded and $\left\{y_{\alpha}\right\}_{\alpha \in \Gamma}$ is a bounded net, it follows that

$$
\limsup _{\alpha}\left[\beta_{0}\left(y_{\alpha}\right)\left(\min _{w \in T\left(y_{\alpha}\right)} R e\left\langle w, y_{\alpha}-x\right\rangle+h\left(y_{\alpha}\right)-h(x)\right)\right]=0 .
$$


Also $\beta_{0}(y)\left[\min _{w \in T(y)} \operatorname{Re}\langle w, y-x\rangle+h(y)-h(x)\right]=0$. Thus

$$
\begin{aligned}
\limsup _{\alpha} & {\left[\beta_{0}\left(y_{\alpha}\right)\left(\min _{w \in T\left(y_{\alpha}\right)} \operatorname{Re}\left\langle w, y_{\alpha}-x\right\rangle+h\left(y_{\alpha}\right)-h(x)\right)\right]+\sum_{p \in E^{*}} \beta_{p}(y) \operatorname{Re}\langle p, y-x\rangle } \\
& \left.=\sum_{p \in E^{*}} \beta_{p}(y) \operatorname{Re}\langle p, y-x\rangle \text { (by }(3.1)\right) \\
& =\beta_{0}(y)\left[\min _{w \in T(y)} \operatorname{Re}\langle w, y-x\rangle+h(y)-h(x)\right]+\sum_{p \in E^{*}} \beta_{p}(y) \operatorname{Re}\langle p, y-x\rangle .
\end{aligned}
$$

For $t=1$ we have $\phi\left(x, y_{\alpha}\right) \leq 0$ for all $\alpha \in \Gamma$, i.e.,

$$
\beta_{0}\left(y_{\alpha}\right)\left[\min _{w \in T\left(y_{\alpha}\right)} R e\left\langle w, y_{\alpha}-x\right\rangle+h\left(y_{\alpha}\right)-h(x)\right]+\sum_{p \in E^{*}} \beta_{p}\left(y_{\alpha}\right) \operatorname{Re}\left\langle p, y_{\alpha}-x\right\rangle \leq 0
$$

for all $\alpha \in \Gamma$. Therefore

$$
\begin{aligned}
& \limsup _{\alpha}\left[\beta_{0}\left(y_{\alpha}\right)\left(\min _{w \in T\left(y_{\alpha}\right)} \operatorname{Re}\left\langle w, y_{\alpha}-x\right\rangle+h\left(y_{\alpha}\right)-h(x)\right)\right] \\
& +\underset{\alpha}{\liminf }\left[\sum_{p \in E^{*}} \beta_{p}\left(y_{\alpha}\right) \operatorname{Re}\left\langle p, y_{\alpha}-x\right\rangle\right] \\
& \leq \limsup _{\alpha}\left[\beta_{0}\left(y_{\alpha}\right)\left(\min _{w \in T\left(y_{\alpha}\right)} R e\left\langle w, y_{\alpha}-x\right\rangle+h\left(y_{\alpha}\right)-h(x)\right)\right. \\
& \left.+\sum_{p \in E^{*}} \beta_{p}\left(y_{\alpha}\right) \operatorname{Re}\left\langle p, y_{\alpha}-x\right\rangle\right] \\
& \leq 0(\text { by }(3.3)) \text {. }
\end{aligned}
$$

Thus

$$
\begin{gathered}
\limsup _{\alpha}\left[\beta_{0}\left(y_{\alpha}\right)\left(\min _{w \in T\left(y_{\alpha}\right)} \operatorname{Re}\left\langle w, y_{\alpha}-x\right\rangle+h\left(y_{\alpha}\right)-h(x)\right)\right] \\
+\sum_{p \in E^{*}} \beta_{p}(y) \operatorname{Re}\langle p, y-x\rangle \leq 0 .
\end{gathered}
$$

Hence by (3.2) and (3.4), we have $\phi(x, y) \leq 0$.

Case 2. $\beta_{0}(y)>0$.

Since $\beta_{0}\left(y_{\alpha}\right) \rightarrow \beta_{0}(y)$, there exists $\lambda \in \Gamma$ such that $\beta_{0}\left(y_{\alpha}\right)>0$ for all $\alpha \geq \lambda$. Then for $t=0$ we have $\phi\left(y, y_{\alpha}\right) \leq 0$ for all $\alpha \in \Gamma$, i.e.,

$$
\beta_{0}\left(y_{\alpha}\right)\left[\min _{w \in T\left(y_{\alpha}\right)} R e\left\langle w, y_{\alpha}-y\right\rangle+h\left(y_{\alpha}\right)-h(y)\right]+\sum_{p \in E^{*}} \beta_{p}\left(y_{\alpha}\right) R e\left\langle p, y_{\alpha}-y\right\rangle \leq 0
$$

for all $\alpha \in \Gamma$. Thus

$$
\begin{gathered}
\limsup _{\alpha}\left[\beta_{0}\left(y_{\alpha}\right)\left(\min _{w \in T\left(y_{\alpha}\right)} \operatorname{Re}\left\langle w, y_{\alpha}-y\right\rangle+h\left(y_{\alpha}\right)-h(y)\right)\right. \\
\left.+\sum_{p \in E^{*}} \beta_{p}\left(y_{\alpha}\right) \operatorname{Re}\left\langle p, y_{\alpha}-y\right\rangle\right] \leq 0 .
\end{gathered}
$$


Hence

$$
\begin{aligned}
& \limsup _{\alpha}\left[\beta_{0}\left(y_{\alpha}\right)\left(\min _{w \in T\left(y_{\alpha}\right)} \operatorname{Re}\left\langle w, y_{\alpha}-y\right\rangle+h\left(y_{\alpha}\right)-h(y)\right)\right] \\
& +\underset{\alpha}{\liminf }\left[\sum_{p \in E^{*}} \beta_{p}\left(y_{\alpha}\right) \operatorname{Re}\left\langle p, y_{\alpha}-y\right\rangle\right] \\
& \leq \limsup _{\alpha}\left[\beta_{0}\left(y_{\alpha}\right)\left(\min _{w \in T\left(y_{\alpha}\right)} \operatorname{Re}\left\langle w, y_{\alpha}-y\right\rangle+h\left(y_{\alpha}\right)-h(y)\right)\right. \\
& \left.+\sum_{p \in E^{*}} \beta_{p}\left(y_{\alpha}\right) \operatorname{Re}\left\langle p, y_{\alpha}-y\right\rangle\right] \\
& \leq 0 \text { (by (3.5)). }
\end{aligned}
$$

Since $\liminf \operatorname{in}_{\alpha}\left[\sum_{p \in E^{*}} \beta_{p}\left(y_{\alpha}\right) \operatorname{Re}\left\langle p, y_{\alpha}-y\right\rangle\right]=0$, we have

$$
\limsup \left[\beta_{0}\left(y_{\alpha}\right)\left(\min _{w \in T\left(y_{\alpha}\right)} \operatorname{Re}\left\langle w, y_{\alpha}-y\right\rangle+h\left(y_{\alpha}\right)-h(y)\right)\right] \leq 0 .
$$

Since $\beta_{0}\left(y_{\alpha}\right)>0$ for all $\alpha \geq \lambda$, it follows that

$$
\begin{aligned}
& \left.\beta_{0}(y) \limsup _{\alpha}\left[\min _{w \in T\left(y_{\alpha}\right)} \operatorname{Re}\left\langle w, y_{\alpha}-y\right\rangle+h\left(y_{\alpha}\right)-h(y)\right)\right] \\
& \quad=\limsup _{\alpha}\left[\beta_{0}\left(y_{\alpha}\right)\left(\min _{w \in T\left(y_{\alpha}\right)} \operatorname{Re}\left\langle w, y_{\alpha}-y\right\rangle+h\left(y_{\alpha}\right)-h(y)\right)\right] .
\end{aligned}
$$

Since $\beta_{0}(y)>0$, by (3.6) and (3.7) we have

$$
\limsup _{\alpha}\left[\min _{w \in T\left(y_{\alpha}\right)} \operatorname{Re}\left\langle w, y_{\alpha}-y\right\rangle+h\left(y_{\alpha}\right)-h(y)\right] \leq 0 .
$$

Since $T$ is $h$-pseudo-monotone, we have

$$
\begin{gathered}
\liminf _{\alpha}\left[\min _{w \in T\left(y_{\alpha}\right)} \operatorname{Re}\left\langle w, y_{\alpha}-x\right\rangle+h\left(y_{\alpha}\right)-h(x)\right] \\
\geq \min _{w \in T(y)} \operatorname{Re}\langle w, y-x\rangle+h(y)-h(x) .
\end{gathered}
$$

Since $\beta_{0}(y)>0$, we have

$$
\begin{gathered}
\beta_{0}(y)\left[\liminf \left(\min _{w \in T\left(y_{\alpha}\right)} \operatorname{Re}\left\langle w, y_{\alpha}-x\right\rangle+h\left(y_{\alpha}\right)-h(x)\right)\right] \\
\geq \beta_{0}(y)\left[\min _{w \in T(y)} \operatorname{Re}\langle w, y-x\rangle+h(y)-h(x)\right] .
\end{gathered}
$$

Thus

$$
\begin{aligned}
& \beta_{0}(y)\left[\liminf _{\alpha}\left(\min _{w \in T\left(y_{\alpha}\right)} \operatorname{Re}\left\langle w, y_{\alpha}-x\right\rangle+h\left(y_{\alpha}\right)-h(x)\right)\right]+\sum_{p \in E^{*}} \beta_{p}(y) \operatorname{Re}\langle p, y-x\rangle \\
& \geq \beta_{0}(y)\left[\min _{w \in T(y)} \operatorname{Re}\langle w, y-x\rangle+h(y)-h(x)\right]+\sum_{p \in E^{*}} \beta_{p}(y) \operatorname{Re}\langle p, y-x\rangle .
\end{aligned}
$$

For $t=1$ we also have $\phi\left(x, y_{\alpha}\right) \leq 0$ for all $\alpha \in \Gamma$, i.e.,

$$
\beta_{0}\left(y_{\alpha}\right)\left[\min _{w \in T\left(y_{\alpha}\right)} \operatorname{Re}\left\langle w, y_{\alpha}-x\right\rangle+h\left(y_{\alpha}\right)-h(x)\right]+\sum_{p \in E^{*}} \beta_{p}\left(y_{\alpha}\right) \operatorname{Re}\left\langle p, y_{\alpha}-x\right\rangle \leq 0
$$


for all $\alpha \in \Gamma$. Therefore

$$
\begin{aligned}
& 0 \geq \liminf _{\alpha} {\left[\beta_{0}\left(y_{\alpha}\right)\left(\min _{w \in T\left(y_{\alpha}\right)} \operatorname{Re}\left\langle w, y_{\alpha}-x\right\rangle+h\left(y_{\alpha}\right)-h(x)\right)\right.} \\
&\left.+\sum_{p \in E^{*}} \beta_{p}\left(y_{\alpha}\right) \operatorname{Re}\left\langle p, y_{\alpha}-x\right\rangle\right] \\
& \geq \underset{\alpha}{\liminf }\left[\beta_{0}\left(y_{\alpha}\right)\left(\min _{w \in T\left(y_{\alpha}\right)} \operatorname{Re}\left\langle w, y_{\alpha}-x\right\rangle+h\left(y_{\alpha}\right)-h(x)\right)\right] \\
& \quad+\liminf _{\alpha}\left[\sum_{p \in E^{*}} \beta_{p}\left(y_{\alpha}\right) \operatorname{Re}\left\langle p, y_{\alpha}-x\right\rangle\right] \\
&=\beta_{0}(y)\left[\liminf _{\alpha}\left(\min _{w \in T\left(y_{\alpha}\right)} \operatorname{Re}\left\langle w, y_{\alpha}-x\right\rangle+h\left(y_{\alpha}\right)-h(x)\right)\right] \\
& \quad+\sum_{p \in E^{*}} \beta_{p}(y) \operatorname{Re}\langle p, y-x\rangle .
\end{aligned}
$$

Consequently, by (3.8) and (3.9), we have $\phi(x, y) \leq 0$.

Now, the rest of the proof of Step 1 is similar to the proofs in Step 1 of Theorem 2.1 and Theorem 3.1 in [6]. Thus Step 1 is proved.

Step 2. There exists a point $\hat{w} \in T(\hat{y})$ such that $\operatorname{Re}\langle\hat{w}, \hat{y}-x\rangle+h(\hat{y})-h(x) \leq$ 0 for all $x \in S(\hat{y})$.

Also the same proof of Step 2 of Theorem 2.1 shows that there exists $\hat{w} \in T(\hat{y})$ such that $R e\langle\hat{w}, \hat{y}-x\rangle+h(\hat{y})-h(x) \leq 0$ for all $x \in S(\hat{y})$.

If $X$ is compact, we obtain the following immediate consequence of Theorem 3.1:

Theorem 3.2. Let $E$ be a locally convex Hausdorff topological vector space, $X$ be a non-empty compact convex subset of $E$ and $h: E \rightarrow \mathbb{R}$ be convex such that $h(X)$ is bounded. Let $S: X \rightarrow 2^{X}$ be upper semicontinuous such that each $S(x)$ is closed convex and $T: X \rightarrow 2^{E^{*}}$ be h-pseudo-monotone and be upper semicontinuous from $\operatorname{co}(A)$ to the weak ${ }^{*}$-topology on $E^{*}$ for each $A \in \mathcal{F}(X)$ such that each $T(x)$ is weak*compact convex and $T(X)$ is strongly bounded. Suppose that the set $\Sigma=\{y \in X$ : $\left.\sup _{x \in S(y)}\left[\inf _{w \in T(y)} \operatorname{Re}\langle w, y-x\rangle+h(y)-h(x)\right]>0\right\}$ is open in $X$. Then there exists $\hat{y} \in X$ such that (i) $\hat{y} \in S(\hat{y})$ and (ii) there exists $\hat{w} \in T(\hat{y})$ with $\operatorname{Re}\langle\hat{w}, \hat{y}-x\rangle \leq h(x)-h(\hat{y})$ for all $x \in S(\hat{y})$.

Note that if the map $S: X \rightarrow 2^{X}$ is, in addition, lower semicontinuous and for each $y \in \Sigma, T$ is upper semicontinuous at $y$ in $X$, then the set $\Sigma$ in Theorem 3.1 is always open in $X$ as can be seen in the proof of the following:

Theorem 3.3. Let $E$ be a locally convex Hausdorff topological vector space, $X$ be a non-empty paracompact convex and bounded subset of $E$ and $h: E \rightarrow \mathbb{R}$ be convex such that $h(X)$ is bounded. Let $S: X \rightarrow 2^{X}$ be continuous such that each $S(x)$ is compact convex and $T: X \rightarrow 2^{E^{*}}$ be h-pseudo-monotone and be upper semicontinuous from $\operatorname{co}(A)$ to the weak $k^{*}$-topology on $E^{*}$ for each $A \in \mathcal{F}(X)$ such that each $T(x)$ is weak ${ }^{*}$-compact convex and $T(X)$ is strongly bounded. Suppose that for each $y \in \Sigma=\left\{y \in X: \sup _{x \in S(y)}\left[\inf _{w \in T(y)} \operatorname{Re}\langle w, y-x\rangle+h(y)-h(x)\right]>0\right\}, T$ is upper semicontinuous at $y$ from the relative topology on $X$ to the strong topology on $E^{*}$. Suppose further that there exist a non-empty compact subset $K$ of $X$ and a point $x_{0} \in X$ such that $x_{0} \in K \cap S(y)$ and $\inf _{w \in T(y)} \operatorname{Re}\left\langle w, y-x_{0}\right\rangle+h(y)-h\left(x_{0}\right)>0$ for all $y \in X \backslash K$. Then there exists $\hat{y} \in K$ such that (i) $\hat{y} \in S(\hat{y})$ and (ii) there exists $\hat{w} \in T(\hat{y})$ with $\operatorname{Re}\langle\hat{w}, \hat{y}-x\rangle \leq h(x)-h(\hat{y})$ for all $x \in S(\hat{y})$. 
Proof. By virtue of Theorem 3.1, we need only show that the set $\Sigma:=\{y \in X$ : $\left.\sup _{x \in S(y)}\left[\inf _{w \in T(y)} \operatorname{Re}\langle w, y-x\rangle+h(y)-h(x)\right]>0\right\}$ is open in $X$.

Now, following the same arguments as in the proofs of Theorem 3.2 in $[6]$ and Theorem 2.3, we can similarly show that the set $\Sigma$ is open in $X$. Hence by Theorem 3.1 the conclusion follows.

If $X$ is compact, we obtain the following immediate consequence of Theorem 3.3:

Theorem 3.4. Let $E$ be a locally convex Hausdorff topological vector space, $X$ be a non-empty compact convex subset of $E$ and $h: E \rightarrow \mathbb{R}$ be convex such that $h(X)$ is bounded. Let $S: X \rightarrow 2^{X}$ be continuous such that each $S(x)$ is closed convex and $T: X \rightarrow 2^{E^{*}}$ be h-pseudo-monotone and be upper semicontinuous from $\operatorname{co}(A)$ to the weak ${ }^{*}$-topology on $E^{*}$ for each $A \in \mathcal{F}(X)$ such that each $T(x)$ is weak $k^{*}$-compact convex and $T(X)$ is strongly bounded. Suppose that for each $y \in \Sigma=\left\{y \in X: \sup _{x \in S(y)}\left[\inf _{w \in T(y)} \operatorname{Re}\langle w, y-x\rangle+h(y)-h(x)\right]>0\right\}, T$ is upper semicontinuous at $y$ from the relative topology on $X$ to the strong topology on $E^{*}$. Then there exists $\hat{y} \in X$ such that (i) $\hat{y} \in S(\hat{y})$ and (ii) there exists $\hat{w} \in T(\hat{y})$ with $\operatorname{Re}\langle\hat{w}, \hat{y}-x\rangle \leq h(x)-h(\hat{y})$ for all $x \in S(\hat{y})$.

We remark here that in Theorems 3.1-3.4, the condition " $h: E \rightarrow \mathbb{R}$ be convex" can be replaced by the condition " $h: X \rightarrow \mathbb{R}$ be convex such that $\left.h\right|_{c o(A)}$ is continuous for each $A \in \mathcal{F}(X)$ ".

\section{REFERENCES}

1. J. P. Aubin, "Applied Functional Analysis", Wiley-Interscience, New York, 1979. MR 81a: 46083

2. A. Bensousson and J. L. Lions, Nouvelle formulation des problèmes de contrôle impulsionnel et applications, C. R. Acad. Sci. 29 (1973), 1189-1192.

3. H. Brézis, L. Nirenberg and G. Stampacchia, A remark on Ky Fan's minimax principle, Bollettino U.M.I. (4) 6 (1972), 293-300. MR 48:2850

4. D. Chan and J. S. Pang, The generalized quasi-variational inequality problem, Math. Oper. Res. 7 (1982), 211-222. MR 83m:49009

5. M. S. R. Chowdhury and K.-K. Tan, Generalization of Ky Fan's minimax inequality with applications to generalized variational inequalities for pseudo-monotone operators and fixed point theorems, J. Math. Anal. and Appl. 204 (1996), 910-929. CMP 97:05

6. M. S. R. Chowdhury and K.-K. Tan, Generalized quasi-variational inequalities for upper semicontinuous operators on non-compact sets, Nonlinear Analysis, Proceedings of the Second World Congress of Nonlinear Analysis, Vol. 30:8 (1997), pp. 5389-5394.

7. J. Dugundji, Topology, Allyn and Bacon, Boston, 1966. MR 33:1824

8. K. Fan, A Minimax Inequality and Applications, in "Inequalities", Vol. III, "Proceedings, Third Symposium on Inequalities"(O. Shisha, Ed.), Academic Press, New York, pp. 103-113, 1972. MR 49:5779

9. H. Kneser, Sur un théoréme fondamental de la théorie des jeux, C. R. Acad. Sci. Paris 234 (1952), 2418-2420. MR 14:301a

10. R. T. Rockafeller, Convex Analysis, Princeton University Press, Princeton, 1970. MR 43:445

11. M.-H. Shih and K.-K. Tan, Generalized quasi-variational inequalities in locally convex topological vector spaces, J. Math. Anal. Appl. 108 (1985), 333-343. MR 86j:90149

12. W. Takahashi, Nonlinear variational inequalities and fixed point theorems, J. Math. Soc. Japan 28 (1976), 166-181. MR 53:3817

Department of Mathematics, Statistics and Computing Science, Dalhousie University, Halifax, Nova Scotia, Canada B3H 3J5

E-mail address: mohammad@mscs.dal.ca

E-mail address: kktan@mscs.dal.ca 